mit hoher Auflösung zu bestimmen. So lässt sich mit CryoSat-2zum Beispiel recht gut erkennen, an welcher Stelle eines Gletschers viel Schnee niedergegangen ist. GRACE-FO tastet ein Raster von 400 Kilometern ab, das ist recht grob. CryoSat-2 hat mit typischerweise fünf Kilometern eine deutlich höhere Auflösung. Doch auch CryoSat-2 hat seine Grenzen. Die Radarstrahlen dringen ein wenig in Schnee und Eis ein, was die exakte Messung der Schnee- oder Eishöhe schwierig macht, vor allem weil man die genauen Bedingungen vor Ort nicht kennt. Das AWI führt deshalb zusätzlich Kalibrationsmessungen mit seinen Forschungsflugzeugen durch. Eine weitere Unsicherheit: Schnee sackt nach und nach unter seinem Gewicht in sich zusammen. Auch das verfälscht die Höhenmessung. $\mathrm{Ob}$ eine Veränderung der Eis- und Schneehöhe durch eine solche Kompaktierung oder durch Schmelze ausgelöst wurde, könne allein mit CryoSat-2-Daten nicht festgestellt werden. Dazu würde

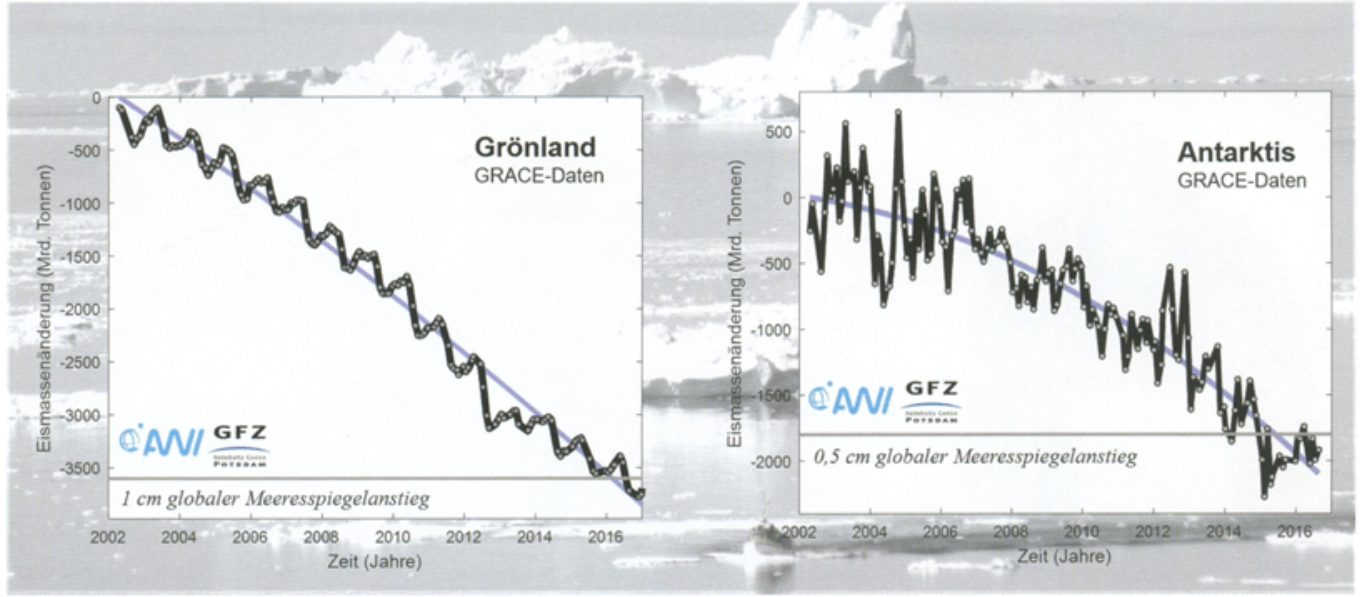

Eismassenänderung in Grönland und der Antarktis aus Daten der GRACE-Mission (2002-2017). Die Einheit ist Mrd. Tonnen, wobei ca. 360 Mrd. Tonnen Eisverlust zu einem globalen mittleren Meeresspiegelanstieg von $1 \mathrm{~mm}$ führen. Die Verluste in Grönland sind gegenwärtig etwa doppelt so hoch wie jene in der Antarktis - 263 Mrd. Tonnen pro Jahr gegenüber 143 Mrd. Tonnen pro Jahr. In Grönland ist der starke Schmelzverlust im Sommer 2012 zu erkennen, als Rekord-Lufttemperaturen erreicht wurden. Der Eismassenverlust in der Antarktis beschleunigt sich signifikant jedes Jahr um 14 Mrd. Tonnen pro Jahr, das liegt am beschleunigten Eisrückzug in der Amundsen See, West Antarktis. (Grafik: GravIS/GFZ)

laut Ingo Sasgen wiederum GRACE Follow-On gebraucht. Denn die Veränderung des Schwerefeldes zeige, ob es tatsächlich Eisund Schneemassenverluste gäbe. Die Daten der verschiedenen Satelliten ergänzen sich also sehr gut, weil jeder seine Stärken hat. Mit dem Start von GRACE-FO schließt sich jetzt nach rund ei- nem Jahr eine Lücke im Feld der Wissenschafts-Satelliten. Wie beim Vorgänger ist die Missionsdauer von GRACE-F0 zunächst für fünf Jahre geplant. Ingo Sasgen hofft, dass die zweite Generation genau wie die erste aber vielleicht sogar 15 Jahre durchhält. Dann würde insgesamt eine

Zeitreihe von rund 30 Jahren zur Internet: www.awi.de

\title{
SGK-Nachrichten
}

\section{Vorstandswahlen der SGK}

Anlässlich der 49. Mitgliederversammlung der Schweizerischen Gesellschaft für Kartografie vom 14.04. 2018 in St. Gallen wurde der gesamte Vorstand turnusgemäss neu gewählt und dabei die Hälfte des Vorstandes neu zusammengesetzt. Der neue Vorstand besteht zudem zum ersten Mal aus drei Frauen und drei Männern.

Nicht mehr zur Wahl angetreten sind die langjährigen Vorstandsmitglieder Stefan Räber (Sekretär, 18 Jahre!), Martin Urech (Beisitzer und ehemaliger Präsident, 11 Jahre) sowie Philipp Marty (Kassier, 6 Jahre), welche herzlich von den
Anwesenden verabschiedet und für ihre Leistungen für die Gesellschaft gewürdigt wurden.

Einstimmig neu in den Vorstand für jeweils drei Jahre gewählt wurden die Mitglieder Anita Bertiller (Kartografin, Sigmaplan Bern), Francis Baca (Kartograf, swisstopo) und Mark Wigley (Geograf und Kartograf, Esri Schweiz). Der Vorstand freut sich über die hochqualifizierten Kandidaturen, heisst die neuen Mitglieder herzlich willkommen und freut sich auf die Zusammenarbeit! Wieder in den Vorstand gewählt wurden auch die bisherigen Mitglieder Madlena Cavelti-Hammer und Susanne Bleisch. Weitere Informationen zum Vorstandswechsel und zur

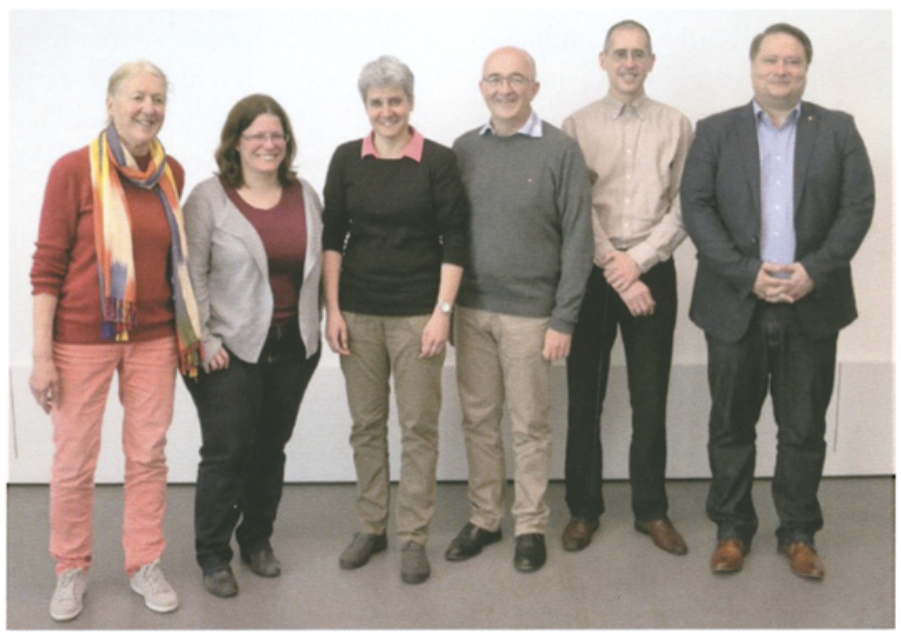

Vorstand 2018, von links: Madlena Cavelti, Anita Bertiller (neu), Susanne Bleisch, Mark Wigley (neu), Francis Baca (neu), Thomas Schulz. (Foto: Stephan Wondrak)

Mitgliederversammlung finden sich auch im SGK-Infoblatt 2/2018: http://kartografie.ch/wpcontent/uploads/2018/04/carto_ news_2018_2_web.pdf und auf der Website der SGK: http://kar tografie.ch/uber-uns/vorstand/

Dr. Thomas Schulz,

Präsident der SGK
Verfügung stehen. Für Klimamodelle wäre das eine wirklich aussagekräftige Zeitspanne. Diese Daten dürften noch in Jahrzehnten für die Klimaforschung von Bedeutung sein.

\section{Informationen:}

Alfred-Wegener-Institut,

Bremerhaven; 